\title{
APLICABILIDADE DO INVENTÁRIO DE BECK NOS ACADÊMICOS DE ENFERMAGEM EM UMA INSTITUIÇÃO DE ENSINO DE MINAS GERAIS
}

Karina de Fátima Rodrigues dos Santos ${ }^{1}$ Valéria Tristão Ferreira², Paula Rogéria da Silva', Débora Vitória Alexandrina Lisboa Vilella ${ }^{1}$

Objetivo: Avaliar a incidência de sinais e sintomas de depressão entre acadêmicos de enfermagem de uma instituição do sul de Minas Gerais. Metodologia: estudo de natureza quantitativa, com abordagem exploratória, descritiva e transversal, com a participação de 116 acadêmicos de enfermagem selecionados entre o primeiro e o décimo período de graduação, avaliados por meio do inventário de Beck. Resultados: dos 21 itens avaliados pelo referido instrumento, evidenciamos que apenas 3 itens que compõem o inventário (autoacusação, inibição do sono e irritabilidade) se destacaram entre a amostra estudada. Conclusão: Por meio da aplicação do inventário de Beck, evidenciamos a probabilidade de se desenvolver entre estes acadêmicos um quadro depressivo futuramente, caso os mesmos não tenham um equilibrio emocional, apoio de professores, familia e comunidade para prevenir a manifestação da depressão.

Descritores: Depressão; Enfermagem; Inventário.

\section{APPLICABILITY OF BECK'S INVENTORY IN ACADEMICS OF NURSING IN A HEALTH INSTITUTION OF THE MINAS GERAIS}

Objective: To evaluate the incidence of signs and symptoms of depression among nursing students from an institution in the south of Minas Gerais. Methodology: a quantitative study, with an exploratory, descriptive and cross-sectional approach, with the participation of 116 nursing students selected between the first and the tenth graduation period, evaluated through the Beck inventory. Results: of the 21 items evaluated by said instrument, we showed that only 3 items that compose the inventory (selfaccusation, sleep inhibition and irritability) stood out among the sample studied. Conclusion: Using the Beck inventory, we show the probability of developing a future depressive state among these students, if they do not have an emotional balance, support of teachers, family and community to prevent the manifestation of depression.

Descriptors: Depression; Nursing; Inventory.

\section{APLICABILIDAD DEL INVENTARIO DE BECK EN LOS ACADÉMICOS DE ENFERMERÍA EN UNA INSTITUCIÓN} DE ENSEÑANZA DE MINAS GERAIS

Objetivo: Evaluar la incidencia de signos y sintomas de depresión entre académicos de enfermeria de una institución del sur de Minas Gerais. Metodología: estudio de la naturaleza cuantitativa, abordaje exploratorio, descripción y transversales, con una participación de 116 académicos de enfermería seleccionados entre el primer y el último periodo de graduación, evaluados por medio del inventario de Beck. Resultados: de los 21 años más tarde que el instrumento recomendado, evidenciamos que sólo 3 items que componen el inventario (autoacusación, inhibición del sueño e irritabilidad) se llevaron entre la muestra estudiada. Conclusión: A través de la aplicación del inventario de Beck, evidenciamos una unión de desarrollo entre empresas académicas en un cuadrúpedo depresivo en el futuro, en caso de que no se haya realizado una prueba emocional, apoyo de profesores, familia y comunidad para prevenir una manifestación de depresión.

Descriptores: Depresión; Enfermería; Inventario. 


\section{INTRODUÇÃO}

A depressão é a doença psiquiátrica mais comum evidenciada na atualidade. Antigamente esta enfermidade era denominada como tristeza, melancolia, entre outras nomenclaturas. Atualmente, sabe-se que sua etiologia se relaciona a duas vertentes; a natureza endógena, devido a presença de alterações na atuação dos neurotransmissores; e à natureza exógena, que remete a presença de fatores ambientais, sendo exemplo dos mesmos: a descoberta de uma doença, a situação de desemprego, o término de um relacionamento, entre outros ${ }^{(1)}$.

A depressão é um transtorno afetivo ao qual se pode observar a redução nos níveis cerebrais das monoaminas, em especial a noradrenalina e a serotonina. Esses neurotransmissores são responsáveis pela estimulação do sistema límbico promovendo, assim, sensação de conforto, prazer e bem-estar ao indivíduo; logo, quando uma pessoa sofre uma perda significativa, como a morte de um filho ou do esposo, a separação de um cônjuge, a perda do emprego, ou é acometida por uma doença grave, ocorre em seu sistema nervoso uma diminuição ou até mesmo ausência dos neurotransmissores referidos, ocasionando um estado de tristeza muito intensa e prolongada, quadro esse que muitas vezes é diagnosticado como depressão mental(2).

Estima-se que 15 a 25\% da população do mundo sofram com esse transtorno, ou seja, em cada quatro pessoas distintas, uma apresentará os sintomas da referida morbidade necessitando de tratamento pelo menos uma vez na vida. As estatísticas apontam que, no Brasil, aproximadamente 24 a 30 milhões de pessoas já apresentaram, apresentam ou vão apresentar pelo menos um episódio depressivo ao longo da vida ${ }^{(3)}$

Atualmente, sabe-se que o estado depressivo afeta a estima pessoal, distorce o modo como as pessoas se enxergam e sua percepção do outro e do mundo. No caso dos alunos de Enfermagem, a depressão pode estar relacionada ao despertar de sentimentos durante o processo de ensinoaprendizagem que envolve relacionamento interpessoal entre companheiros de sala de aula, professores e paciente ${ }^{(3)}$.

É durante o ensino clínico que se manifesta nos acadêmicos sentimento de insegurança e medo, haja vista que estes futuros profissionais são cobrados quanto a manter postura profissional adequada, ter conhecimentos e habilidades para avançar no curso escolhido e assim alcançarem o diploma tão esperado. Algumas das situações que ilustram bem a presença desses sentimentos no cotidiano acadêmico são evidenciadas quando o aluno apresenta dificuldade de interação com o paciente a ser cuidado, não havendo uma comunicação adequada entre paciente-aluno. Outra situação comum a ser citada é a manifestação do sentimento de medo ao lidar com o paciente, haja vista que o acadêmico receia causar dano durante o cuidado prestado ao paciente, ou mesmo prejudicá-lo devido à pouca habilidade técnica e conhecimentos limitado(4).

Logo, em virtude da promoção de boa saúde mental para o acadêmico e futuro profissional de enfermagem, fica evidente que os docentes necessitam desenvolver não só ensino de qualidade para estes alunos, mas também, desempenhar suas atividades docentes atentando-se para a presença de possíveis manifestações de sinais e sintomas depressivos entre os acadêmicos. Sabe-se que quando as manifestações de depressão entre acadêmicos é evidenciada, o docente pode trabalhar com estes alunos por meio de ações e intervenções que venham a reduzir os sintomas apresentados, evitando assim, agravo maiores na vida deste estudante fazendo com que o mesmo torne-se um bom profissional que oferta os melhores cuidados aos pacientes ${ }^{(3)}$.

Pautados na realidade descrita nos parágrafos acima, este estudo tem como objetivo avaliar a incidência de sinais e sintomas de depressão entre acadêmicos de enfermagem de uma instituição do sul de Minas Gerais.

\section{METODOLOGIA}

Estudo de natureza quantitativa, como abordagem exploratória, descritiva e transversal realizado na cidade de Itajubá Minas Gerais. No ano de 2015, ano ao qual foi realizada a coleta de dados para o presente estudo, o curso de graduação em enfermagem da Escola de Enfermagem Wenceslau Braz contava com um total de 193 alunos matriculados. Para a definição da amostra foram aplicados 116 questionários em acadêmicos que cursavam do 1 으 ao 10 o período de graduação do curso referido, que correspondessem ao perfil desejado pelos pesquisadores, logo, participaram do estudo um percentual de $60 \%$ dos alunos matriculados no curso.

A coleta de dados ocorreu entre o primeiro e o segundo semestre do ano de 2015, nos meses de março a agosto do respectivo ano. Aos acadêmicos selecionados, foi aplicado o instrumento para avaliação da presença de fatores de risco para depressão denominado inventário de Beck, este instrumento compreende um questionário que tem como objetivo fazer a autoavaliação do indivíduo quanto a sua probabilidade para desenvolver depressão nos próximos anos.

O Inventário de Back foi desenvolvido no anos de 1971 pelo Centro de Terapia Cognitiva (Center for Cognitive Therapy - CCT) da Escola Médica da Universidade da Pensilvânia; no Brasil, este instrumento foi validado no ano de 1998 e considerado como um instrumento de domínio público. Esse questionário tem sido muito utilizado para avaliar as cognições associadas à depressão, em pacientes 
psiquiátricos e indivíduos que não possuem enfermidades mentais, sendo um dos mais aceitos no que diz respeito a avaliação da intensidade de depressão, tendo um ótimo desempenho nesse quesito(5).

O referido instrumento é composto por 21 itens mesclados entre sintomas e atitudes em quatro graus de intensidade, sendo os mesmos: tristeza, pessimismo, sensação de fracasso, falta de satisfação, sensação de culpa, sensação de punição, autodepreciação, autoacusação, ideias suicidas, crises de choro, irritabilidade, retração social, indecisão, distorção de imagem corporal, inibição do trabalho, distúrbios do sono, fadiga, perda de apetite, perca de peso, preocupação somática, diminuição de libido. Cada item engloba quatro afirmativas (0, 1, 2 ou 3). Os participantes podem marcar mais de uma resposta em cada questão do inventário, porém, durante a análise do respectivo questionário será levado em consideração a alternativa de maior valor assinalada pelo participante em cada questão(5).

Com o intuito de favorecer tempo hábil para os participantes, bem como conforto e comodidade frente a sua participação, os questionários foram distribuidos entre os participantes que apresentaram o perfil desejado pelas pesquisadoras, e estes levaram o questionário para sua casa sendo estipulado o prazo de sete dias para a devolução do mesmo aos respectivos pesquisadores.

Como critérios de inclusão, adotamos selecionar acadêmicos de enfermagem com mais de 18 anos; que estivessem regularmente matriculado na EEWB e aceitassem participar do estudo. Logo, como critérios de exclusão, os candidatos que não contemplaram o perfil supra citado foram automaticamente excluídos da amostra final.
Os dados coletados por meio de um questionário com as respostas dos participantes foram analisados onde consideramos medidas de tendência central como a frequência absoluta e relativa e interpretados por meio da escala de pontos, conforme recomenda o IDB: menos de 10: depressão mínima; de 10 a 18: depressão leve a moderada; de 19 a 29: depressão moderada a grave; e de 30 a 63: depressão grave.

O estudo respeitou os preceitos éticos presentes na resolução 466/12 que trata da ética em pesquisa envolvendo seres humanos. $O$ estudo foi encaminhado para o Comitê de Ética em Pesquisa da Escola de Enfermagem Wenceslau Braz sendo aprovado com número de parecer consubstanciado $\mathrm{n}$ ㅇ 497.700 .

\section{RESULTADOS}

Dos 21 itens avaliados pelo inventário de Beck, aplicado no estudo, apenas 3 itens foram evidenciados dentre os 21 itens do respectivo instrumento, se mostraram promissores frente à avaliação da depressão em acadêmicos de enfermagem. Os itens mencionados foram: distúrbio sono, irritabilidade e autoacusação.

No que se refere ao item distúrbio do sono, observou-se uma maior incidência no item "não durmo tão bem como costumava, fato este representado por um percentual de $58,62 \%$; no tocante irritabilidade, observou-se que $47,41 \%$ afirmam se sentirem mais aborrecidos (a) ou irritados (a) do que antes e finalizando, no item autoacusação, observouse que $51,72 \%$ se criticam por apresentarem momentos de fraquezas e cometerem erros. A tabela abaixo ilustra a avaliação dos itens supra citados:

Tabela 1 - Inventário de Beck, Itajubá-MG (N=116)

\begin{tabular}{|c|c|c|}
\hline Variáveis & $\begin{array}{c}\text { Frequência } \\
\text { Absoluta }\end{array}$ & $\begin{array}{c}\text { Frequência } \\
\text { Relativa }\end{array}$ \\
\hline \multicolumn{3}{|l|}{ 2. PESSIMISMO } \\
\hline 1) Sinto-me desencorajado(a) em relação ao futuro. & 23 & $19,8 \%$ \\
\hline 2) Sinto que nada tenho a esperar. & 1 & $0,9 \%$ \\
\hline 3) Sinto que o futuro é sem esperança e que as coisas não podem melhorar. & 2 & $1,7 \%$ \\
\hline 0) Não me sinto fracassado (a). & 88 & $75,9 \%$ \\
\hline 1) Sinto que falhei mais do que um individuo médio. & 22 & $19 \%$ \\
\hline 2) Quando analiso a minha vida passada, tudo o que vejo é uma quantidade de fracassos. & 6 & $5,1 \%$ \\
\hline 3) Sinto que sou um completo fracasso. & 0 & $0 \%$ \\
\hline \multicolumn{3}{|l|}{ 4. FALTA DE SATISFAÇÃO } \\
\hline
\end{tabular}


Tabela 1 - Inventário de Beck, Itajubá-MG (N=116) (continuação)

\begin{tabular}{|c|c|c|}
\hline Variáveis & $\begin{array}{c}\text { Frequência } \\
\text { Absoluta }\end{array}$ & $\begin{array}{c}\text { Frequência } \\
\text { Relativa }\end{array}$ \\
\hline \multicolumn{3}{|l|}{ 5.SENSAÇÃO DE CULPA } \\
\hline 1) Sinto-me culpado(a) grande parte do tempo. & 24 & $20,69 \%$ \\
\hline 2) Sinto-me bastante culpado(a) a maior parte do tempo. & 1 & $0,87 \%$ \\
\hline 3) Sinto-me culpado(a) durante o tempo todo. & 0 & $0 \%$ \\
\hline 0) Não me sinto que esteja a ser punido(a). & 92 & $79,31 \%$ \\
\hline 1) Sinto que posso ser punido(a). & 21 & $18,10 \%$ \\
\hline 2) Sinto que mereço ser punido(a). & 2 & $1,72 \%$ \\
\hline 3) Sinto que estou a ser punido(a). & 1 & $0,87 \%$ \\
\hline \multicolumn{3}{|l|}{ 7. AUTODEPRIAÇÃO } \\
\hline
\end{tabular}

8. AUTOACUSAÇÃO

0) Não sinto que eu seja pior que qualquer outra pessoa. $\quad 46 \quad 39,66 \%$

1) Critico-me pelas minhas fraquezas ou erros.

$60 \quad 51,72 \%$

2) Culpo-me constantemente pelas minhas faltas.

3) Culpo-me de todas as coisas más que acontecem.

$8 \quad 6,90 \%$

9. IDEIAS SUICIDAS

0) Não tenho qualquer ideia de me matar.

1) Tenho ideias de me matar, mas não sou capaz de as concretizar.

2) Gostaria de me matar.

3) Matar-me-ia se tivesse uma oportunidade.

$21,72 \%$

\section{CRISE DE CHORO}

0) Não costumo chorar mais do que o habitual.

$91,38 \%$

1) Choro mais agora do que costumava fazer.

$\begin{array}{cc}106 & 91,38 \% \\ 8 & 6,90 \% \\ 2 & 1,72 \% \\ 0 & 0 \%\end{array}$

2) Atualmente, choro o tempo todo.

3) Eu costumava conseguir chorar, mas agora não consigo, ainda que queira.

$\begin{array}{cc}71 & 61,20 \% \\ 32 & 27,59 \% \\ 4 & 3,44 \% \\ 9 & 7,76 \%\end{array}$

\section{IRRITABILIDADE}

0) Não me irrito mais do que costumava.

$31 \quad 26,72 \%$

1) Fico aborrecido(a) ou irritado(a) mais facilmente do que costumava.

2) Atualmente, sinto-me permanentemente irritado(a).

$55 \quad 47,41 \%$

3) Já não consigo ficar irritado(a) com as coisas que antes me irritavam.

$20,69 \%$

\section{RETRAÇÃO SOCIAL}

0) Não perdi o interesse nas outras pessoas.

$5,18 \%$

1) Interesso-me menos do que costumava pelas outras pessoas.

2) Perdi a maior parte do meu interesse nas outras pessoas.

3) Perdi todo o meu interesse nas outras pessoas.

$\begin{array}{cc}71 & 61,20 \% \\ 37 & 31,90 \% \\ 8 & 6,90 \% \\ 0 & 0 \%\end{array}$

13. INDECISÃO

0) Tomo decisões como antes.

1) Adio as minhas decisões mais do que costumava.

2) Tenho mãos dificuldade em tomar decisões do que antes.

3) Já não consigo tomar qualquer decisão.

\section{FADIGA}

0) Não fico mais cansado(a) do que o habitual.

1) Fico cansado(a) com mais dificuldade do que antes.

2) Fico cansado(a) ao fazer quase tudo.

3) Estou demasiado cansado(a) para fazer qualquer coisa.

$\begin{array}{cc}43 & 37,07 \% \\ 40 & 34,49 \% \\ 28 & 24,13 \% \\ 5 & 4,13 \%\end{array}$


Tabela 1 - Inventário de Beck, Itajubá-MG (N=116) (continuação)

\begin{tabular}{|c|c|c|}
\hline Variáveis & $\begin{array}{c}\text { Frequência } \\
\text { Absoluta }\end{array}$ & $\begin{array}{c}\text { Frequência } \\
\text { Relativa }\end{array}$ \\
\hline \multicolumn{3}{|l|}{ 15. DISTORÇÃO DA IMAGEM CORPORAL } \\
\hline 0) Não sinto que a minha aparência seja pior do que costumava ser. & 86 & $74,13 \%$ \\
\hline 1) Preocupo-me porque estou a parecer velho(a) ou nada atraente. & 18 & $15,51 \%$ \\
\hline 2) Sinto que há mudanças permanentes na minha aparência que me tornam nada atraente. & 8 & $6,90 \%$ \\
\hline 3) Considero-me feio(a). & 4 & $3,44 \%$ \\
\hline \multicolumn{3}{|l|}{ 16. INIBIÇÃO DE TRABALHO } \\
\hline 0) Não sou capaz de trabalhar tão bem como antes. & 64 & $55,18 \%$ \\
\hline 1) Preciso de um esforço extra para começar qualquer coisa. & 48 & $37,07 \%$ \\
\hline 2) Tenho que me forçar muito para fazer qualquer coisa. & 8 & $6,90 \%$ \\
\hline 3) Não consigo fazer algum trabalho. & 1 & $0,87 \%$ \\
\hline \multicolumn{3}{|l|}{ 17. DISTURBIO DO SONO } \\
\hline 0) Durmo tão bem como habitualmente. & 38 & $32,76 \%$ \\
\hline 1) Não durmo tão bem como costumava. & 68 & $58,62 \%$ \\
\hline \multicolumn{3}{|l|}{ 2) Acordo 1 ou 2 horas antes que o habitual e tenho dificuldade em voltar a adormecer. } \\
\hline \multirow[t]{2}{*}{ 3) Acordo várias vezes mais cedo do que costumava e não consigo voltar a dormir. } & 7 & $6,03 \%$ \\
\hline & 3 & $2,59 \%$ \\
\hline
\end{tabular}

\section{PERDA DE APETITE}

0) O meu apetite é o mesmo de sempre.

$41,37 \%$

1) Não tenho tanto apetite como costumava ter.

2) O meu apetite, agora, está muito pior. $20,69 \%$

3) Perdi completamente o apetite. $4,31 \%$

\section{PERDA DE PESO}

0) Não perdi muito peso, se é que perdi algum ultimamente.

1) Perdi mais de $2,5 \mathrm{~kg}$.

2) Perdi mais de $5 \mathrm{~kg}$.

3) Perdi mais de 7,5 kg. Estou propositado a tentar perder peso, comendo menos.

Sim Não

$21,72 \%$

\section{PREOCUPAÇÃO SOMÁTICA}

0) A minha saúde não me preocupa mais do que o habitual.

1) Preocupo-me com problemas físicos, como dores e aflições, má-disposição do estômago ou prisão de ventre.

2) Estou muito preocupado(a) com problemas físicos e torna-se difícil pensar em outra coisa.

3) Estou tão preocupado(a) com os meus problemas físicos que não consigo pensar em qualquer outra coisa.

\section{DIMINUIÇÃO DO LIBIDO}

0) Não tenho observado qualquer alteração recente no meu interesse sexual.

$$
5
$$

1) Estou menos interessado(a) na vida sexual do que costumava.

2) Sinto-me, atualmente, muito menos interessado(a) pela vida sexual.

3) Perdi completamente o interesse na vida sexual.

$\begin{array}{cc}96 & 82,76 \% \\ 15 & 12,93 \% \\ 4 & 3,44 \% \\ 1 & 0,87 \%\end{array}$

Nota: Instruções: Neste questionário existem grupos de afirmações. Por favor, leia cuidadosamente cada uma delas. A seguir, selecione a afirmação, em cada grupo, que melhor descreve como se sentiu na semana que passou, incluindo o dia de hoje, assinale entre as alternativas abaixo qual delas representa melhor como você se sente. 
No que se refere a avaliação dos participantes quanto ao grau de depressão, evidenciou-se que 71 alunos apresentaram depressão leve, 33 depressão leve a moderada,
11 depressão moderada a grave e 1 aluno depressão grave. Abaixo segue a tabela que ilustrativa:

Tabela 2 - Avaliação do grau de depressão de acadêmicos de enfermagem, Itajubá-MG, (n: 116)

\begin{tabular}{lccccc}
\multicolumn{1}{c}{ Grau de depressão } & № & Média & D. P. & Valor Máximo & Valor Minimo \\
\hline Depressão leve (menos de 10 pts) & 71 & 5,36 & 2,16 & 9 & 2 \\
\hline Depressão leve/moderada (10 a 18 pts) & 33 & 15,04 & 2,65 & 18 & 7 \\
\hline Depressão moderada/grave (19 a 29) & 11 & 22,09 & 3,11 & 28 & 18 \\
\hline Depressão grave (30 a 63) & 1 & 59 & - & 59 & 59 \\
\hline
\end{tabular}

\section{DISCUSSÃO}

As transições vivenciadas pelos acadêmicos durante a vida constituem sempre uma experiência significativa para qualquer indivíduo, haja vista que a vida universitária representa, para muitos destes jovens, oportunidade de sair da casa, se desligar da família e vivenciar atividades diferentes daquelas já vividas na escola secundária ${ }^{(4)}$. $O$ ingresso em uma universidade, geralmente, ocorre quando os jovens encontram-se no fim da adolescência e início da vida adulta; logo, no decorrer deste processo, torna-se comum que esse indivíduo apresente momentos de dúvidas e incertezas até que aprenda a conviver com o novo estilo de vida por ele escolhido(b).

Frente à nova realidade dos acadêmicos, fica fácil compreender sua vulnerabilidade para desenvolver quadros de depressão; sendo assim, esses acadêmicos podem vir a manifestar diminuição no seu rendimento escolar. Todavia, quase sempre essa manifestação, entre outras, passa despercebida ou é negligenciada, fazendo com que o mesmo conviva com os sintomas depressivos por anos sem procurar orientação profissional, caracterizando a sintomatologia como algo natural, que faz parte de seu cotidiano e de sua personalidade ${ }^{(7)}$.

Os acadêmicos tendem a camuflar esta situação por muito tempo até que a mesma se torne crítica, ou seja, quando são relatadas pelo aluno manifestações clínicas como gastrite, perda de peso e transtornos alimentares, insônia, estresse, irritabilidade, autoacusação entre outros. Somente quando se fazem presentes os agravos de âmbito físicos, é que esses jovens decidem então buscar auxílio de profissionais de saúde ${ }^{(7)}$.

Cerca de $15 \%$ a $25 \%$ dos universitários desenvolvem algum transtorno mental durante o período de formação acadêmica, sendo a depressão a mais comum entre a população citada. Dentre os fatores que justificam a manifestação de depressão durante a graduação são muitos, todavia, os mais citados pela literatura são: extensa carga horária, a falta de tempo para o lazer, a pressão para excelência, a saída da casa dos pais para a entrada em uma Universidade, seguida da mudança para um âmbito não familiar, o afastamento da família, dificuldades financeiras, novo ambiente interpessoal, pressão e preocupações com o futuro e a sobrecarga de trabalho ${ }^{(8,9)}$

Existem diferenças significativas na ocorrência de depressão conforme o período cursado, sendo que a tendência é se agravar com o avançar do curso, fato este compreendido pelas mudanças no estilo de vida, ao longo da graduação, possíveis decepções em relação à formação e pela tensão relacionada ao mercado de trabalho(9).

Embora tenha crescido o ingresso de acadêmicos do sexo masculino na área da enfermagem, a profissão continua sendo representada em sua maioria pela população feminina; fato este que nos ajuda a compreender o item irritabilidade como fator em destaque ao avaliar a depressão em acadêmicos de enfermagem. Os niveis de depressão em relação ao sexo feminino são eventualmente maiores do que nos homens, uma vez que, as mulheres tem mais alterações no estado de humor por serem constantemente influenciadas pelas alterações hormonais provenientes do sexo feminino(4.9). Outra vertente que contribui com a afirmativa está voltada à existência de fatores sociais, como cobranças para execução de múltiplos papeis como maternidade, demanda domiciliar e o ambiente de trabalho.

A mulher são mais vulnerabilidade ao desgaste psicológico, elas são mais críticas frente ao que pensam e ao que sentem, diferente dos homens que tem maior dificuldade 
para expressar os sentimentos que os acometem e por isso dão menor importância para os problemas em si, se comparados as mulheres ${ }^{(9)}$.

A irritabilidade evidenciada entre os acadêmicos de enfermagem participantes do presente estudo pode estar associada ao fim do semestre. Neste período, é comum que os alunos de forma geral demonstram maior nível de irritabilidade, mais facilmente do que de costume, pois à grande quantidade de provas a serem realizadas e demais atividades como seminários, sendo que em sua maioria a pontuação atribuida pode definir a sua nota final na disciplina, comprometendo assim a aprovação destes acadêmicos $^{(10)}$

No que se refere ao item autoacusação, fica fácil identificar o quando a inserção do acadêmico neste contexto de dúvidas, incertezas e pressões diárias influencia na apresentação de atitudes de autoacusação. Durante a graduação, os alunos têm tendência a competir entre si expondo as suas ideias, com a finalidade de serem melhores a cada $\mathrm{dia}^{(11)}$. Logo, o aluno tende a não medir esforços para corresponder às exigências que lhe são impostas, todavia, em algumas situações o cansaço impede seu sucesso e o sentimento de fracasso pode direcioná-lo a manifestar sentimentos depressivos.

No tocante aos distúrbios do sono é natural que os acadêmicos envolvidos em tantas situações de conflito e ansiedade venham a desenvolver um padrão de sono ineficaz a suas necessidades habituais. Os estudantes de forma geral apresentam não só distúrbios do sono de forma isolada, mais associados a outros sintomas como: fadiga, cansaço, falhas de memória, dificuldade de atenção e concentração, hipersensibilidade para sons e luz e alterações do humor. Sabe-se que a exposição a níveis constantes e significativos de pressão frente às exigências acadêmicas alteram os padrões de sono dos alunos, ocasionando duração insuficiente do sono, início do sono atrasado e ocorrência de episódios de cochilos durante o dia(8).

Cabe ressaltar que o indivíduo com depressão, seja ele acadêmico ou não de algum curso de graduação principalmente na área da saúde, geralmente perde interesse pelas atividades diárias, não dorme bem, não tem apetite, sendo comum o surgimento de pensamentos ruins, como ideia de culpa e, nos casos mais graves, pode ocorrer ideias suicidas ou suicídio(3)

No que diz respeito a avaliação final dos participantes do presente estudo, pode-se observar que em sua maioria, os acadêmicos apresentam grau de depressão mínima; este fato pode ser compreendido pelas autoras devi- do à maior parcela dos colaboradores do presente estudo pertencerem ao primeiro e segundo período de graduação em enfermagem, logo, encontram-se no início de sua vida acadêmica e estando eufóricos pelas novidades que o curso pode-lhes proporcionar, estão vivenciando este período de adaptação à vida acadêmica e por isso não assumem tantas responsabilidades como os acadêmicos do quinto período em diante.

Atualmente, sabe-se por meio da diversidade de estudos envolvendo as vertentes depressão e vida acadêmica que devido às incontáveis responsabilidades atribuidas aos acadêmicos, uma grande parcela destes universitários passa a se preocupar de forma exagerada em todas as atividades a serem realizadas e começa a pensar que não será capaz de corresponder a demanda de tarefas propostas, promovendo um grande agastamento emocional na vida do estudante ${ }^{(12)}$.

No estudo realizado por Souza, Tavares e Pinto(13), os autores evidenciaram por meio da amostra estudada que a experiência psicológica dos estudantes varia significativamente ao longo dos últimos anos de curso, havendo mudanças no que foi descrito como fases psicológicas, sendo elas: a) euforia inicial, devido ao ingresso na faculdade; b) decepção, marcada pela cobrança intensa, desempenho dificultoso; c) adaptação e preocupação, decorrentes do período do internato, e a competição pelas vagas de emprego. Logo, pode-se afirmar que há um aumento gradativo a cada ano que se passa na vida do acadêmico, sendo que os níveis de ansiedade, estresse e burnout, os quais culminam principalmente no período de conclusão de curso.

\section{CONCLUSÃO}

Por meio do presente estudo foi possivel observar que o acometimento dos acadêmicos de enfermagem não está relacionado a um fator específico, mas sim a um gama de estressores que se não forem evidenciados de forma precoce podem comprometer seriamente a vida do acadêmico, colocando em risco sua vida profissional e possibilidades almejadas pelo aluno acometido.

É importante ressaltar que, na maioria dos casos, o aluno é o último a identificar o estágio depressivo em que se encontra. Fato este que vem a alertar professores e profissionais da área da educação a manterem-se alertas para promover, de forma precoce, o atendimento e assistência digna aos alunos em quadro depressivo, haja vista que muitos residem em lugares distantes, estando afastados dos familiares que poderiam thes prestar apoio. 
Salienta-se neste estudo a importância de se implantar atendimento de saúde para a clientela estudada, contemplando apoio psicológico para depressão e outros tipos de transtorno mental de forma preventiva e reparatíva quando necessário. Acredita-se que o aporte fornecido aos acadêmicos irá servir como linha guia para auxiliar estes estudantes a lidarem melhor com as mudanças da vida diária não só no momento acadêmico, mas por todo o seu ciclo vitalício.

\section{FINANCIAMENTO}

Manuscrito resultante da Pesquisa original de Iniciação Científica, financiada pela Fundação de Amparo à Pesquisa do Estado de Minas Gerais.

\section{REFERÊNCIAS}

1. Associação Brasileira de Familiares, Amigos e Portadores de Transtornos Afetivos. Transtorno do humor: transtorno bipolar do humo [Internet]. São Paulo; Abrata; 2014 [cited 2017 Abr 27]. Available from: http://abrata.org.br/blogabrata/?p=1487

2. Guyton AC, Hall JE. Tratado de fisiologia médica. 13a ed. Rio de Janeiro: Elsevier; 2017.

3. Camargo RM, Sousa CO, Oliveira MLC. Prevalência de casos de depressão em acadêmicos de enfermagem em uma instituição de ensino de Brasilia. REME [Internet]. 2014 [cited 2017 Mar 30];18(2):392-7. Available from: http://www.reme.org.br/artigo/detalhes/935

4. Vizzoto MM, Martins AC, Jesus SN. Saudades de casa: indicativos de depressão, ansiedade, qualidade de vida e adaptação de estudantes universitários. Rev Psicol. Saúde [Internet]. 2017 [cited 2017 Abr 23];9(1):5973. Available from: http://pepsic.bvsalud.org/pdf/rpsaude/v9nl/v9nla04. pdf

5. Vargas D, Dias APV. Prevalência de depressão em trabalhadores de enfermagem de Unidade de Terapia Intensiva: estudo em hospitais de uma cidade do noroeste do Estado São Paulo. Rev Latino-Am Enfermagem [Internet]. 2011 [cited 2017 Mar 10];19(5):1114-21. Available from: http://www.scielo.br/pdf/rlae/v19n5/pt_08.pdf

6. Ferreira CMG, Kluthcovsky ACGC, Dornelles CF, Stumpf MAM Cordeiro TMG. Qualidade do sono em estudantes de medicina de uma universidade do Sul do Brasil. Conexão Ci. 2017:12(1):78-85.

7. Lima LM, Rodrigues BA, Fontoura H.S. O uso da auriculoterapia no controle da sintomatologia da depressão em acadêmicos da ESEFFEGO. Anais do III Congresso de Ensino, Pesquisa e Extensão da UEG: inovação: inclusão social e direitos; 2016 Out. 19-21; Pirenópolis: UEG; 2016.
8. Cremasco GS, Baptista MN. Depressão, motivos para viver e o significado do suicidio em graduandos do curso de psicologia. Estud. Interdiscip. Psicol [Internet]. 2017 [cited 2017 Fev 15]:8(1):22-37. Available from: http://www.uel.br/revistas/uel/index.php/eip/article/ view/24293

9. Oliveira GS, Rocha CA, Santos BEF, Sena IS, Favaro L, Guerreiro MC. Prevalência e fatores associados à depressão em estudantes de medicina da Universidade Federal do Amapá. Rev. Med. Saúde [Internet]. 2016 [cited 2017 Mar 18];5(3):186-99. Available from: https:// portalrevistas.ucb.br/index.php/rmsbr/article/view/7359

10. Araújo AHI. Relação entre ansiedade/depressão e sobrepeso/ obesidade em estudantes da universidade de Brasília [monografia]. Ceilândia: Universidade de Brasilia; 2015 [cited 2017 Jun 11]. Available from: http://bdm.unb.br/handle/10483/11542

11. Claudino J, Cordeiro R. Niveis de ansiedade e depressão nos alunos do curso de licenciatura em enfermagem o caso particular dos alunos da escola superior de saúde de Portalegre. Educ. Cienc. Tecnol. 2011;32(11):197-210. Available from:

12. Sousa TBO, Ivo EB, Oliveira MM, Araújo DDC, Batista JMM. Depressão e ansiedade na vida acadêmica. Unicatólica. 2017 [cited 2018 Mar 19]:4(2):1-1. Available from: http://publicacoesacademicas. fcrs.edu.br/index.php/mostracientificafarmacia/article/view/1986

13. Souza AS, Tavares KM, Pinto PSP. Depressão em estudantes de medicina: uma revisão sistemática de literatura [Internet]. In: XVI Seminário Estudantil de Produção Acadêmica. Salvador: UNIFACS; 2017 [cited 2018 Mar 20]. p. 1-17. Available from: http://www.revistas. unifacs.br/index.php/sepa/article/viewFile/4815/3318 